\title{
A Delayed Chemostat Model with Impulsive Diffusion and Input on Nutrients
}

\author{
Jianjun Jiao and Shaohong Cai \\ Guizhou Key Laboratory of Economic System Simulation, School of Mathematics and Statistics, \\ Guizhou College of Finance and Economics, Guiyang 550004, China \\ Correspondence should be addressed to Jianjun Jiao, jiaojianjun05@126.com
}

Received 21 August 2009; Revised 2 November 2009; Accepted 30 November 2009

Recommended by Binggen Zhang

A chemostat model with delayed response in growth and impulsive diffusion and input on nutrients is considered. Using the discrete dynamical system determined by the stroboscopic map, we obtain a microorganism-extinction periodic solution. Further, it is globally attractive. The permanent condition of the investigated system is also obtained by the theory on impulsive delay differential equation. Finally, numerical analysis is inserted to illustrate dynamical behaviors of the chemostat system. Our results reveal that the impulsive input amount of nutrients plays an important role on the outcome of the chemostat. Our results provide strategy basis for biochemical reaction management.

Copyright ( 2009 J. Jiao and S. Cai. This is an open access article distributed under the Creative Commons Attribution License, which permits unrestricted use, distribution, and reproduction in any medium, provided the original work is properly cited.

\section{Introduction}

The theory of impulsive differential equation appears as a natural description of several real processes subject to certain perturbations whose duration is negligible in comparison with the duration of the process. Recently, equations of this kind are found in almost every domain of applied science. Many examples are given in [1]. Some impulsive differential equations have been recently introduced in population dynamics[2-7], pulse vaccination [8-11], and chemostat dynamics [12-15].

The chemostat is a basic piece of laboratory apparatus. The advantages are that certain of the biological parameters assumed to influence the outcomes can be controlled by the experimenters. The chemostat plays an important role in bioprocessing, such as ecology, microbiology, chemical engineering, and so forth. Smith and Waltman had made discussion about the chemostat model in [16]. The models contain discrete time delays which account for time which laps between uptaked of nutrient and the assimilation of nutrient into viable biomass. Smith, Waltman [17] and Kuang [18] discussed various aspects of models with 
discrete time delay. Freedman, So, and Waltman[19] were the first to incorporate time delay in chemostat models. Ellermeyer [20], Hsu et al. [21, 22], analyzed a discrete time delay model with two competitive microorganisms for a single nutrient in a chemostat. Hale, Somolinos [23], Hsu et al. [24], Wolkowicz, Zhao [25] investigated competition in chemostat. Ellermeyer et al. [26] did a theoretical and empirical investigation of delayed growth response in the continuous culture of bacteria. Delays occur naturally in biological system by two obvious sources of delays: delays due to the cell cycle; and delays due to the possibility the organism stores the nutrient. Delays appear in a chemostat model in Bush and Cook [27]. They have investigated a model of growth of one organism in the chemostat with a delay in the intrinsic growth rate of the microorganism but with no delay in the substrate equation.

In chemostat's simplest form, the system approximates conditions for plankton growth in lakes, where the limiting nutrients such as silica and phosphate are supplied from streams draining the watershed. In the lakes, the plankton population movements are subject to many factors, such as currents and turbulent lateral diffusion (cf. Levin and Segel [28], Mimura [29], Okubo [30], Freedman and Ruan [31]). Ruan [32] proposed a diffusive plankton-nutrient interaction model with delayed nutrient recycling and delayed growth response and studied Turing instability and the existence of travelling wave solutions. However, they did not investigate a chemostat model with delayed response in growth and impulsive diffusion on nutrients. Many researchers indicated that it was important to consider models with periodic perturbations, since these models may be quite naturally exposed in many real world phenomena, for instance, food supply, mating habits, cross flooding in rainstorm season. In fact, the perturbations such as cross flooding in rainstorm which are not suitable to be considered with continuity. These perturbations bring sudden changes to the system. Systems with sudden perturbations are involving an impulsive differential equations which have been studied intensively and systematically in [1,2]. There are few papers $[8,12,33,34]$ research the chemostat model with impulsive perturbations on nutrients. In this paper, a chemostat model with delayed response in growth and impulsive diffusion on nutrients is investigated, we will obtain a microorganism-extinction periodic solution. Further, it is globally attractive. The permanent condition of the investigated system is also obtained.

The organizations of the paper are as following. In Section 2, we introduce a chemostat model with delayed response in growth and impulsive diffusion on nutrients. In Section 3, we present some preliminary results about the investigated model. Our main results are stated and proven in Section 4. Finally, the numerical analysis is inserted to illustrate the results, and conclude with a brief discussion in Section 5 .

\section{The Model}

We investigate the following chemostat model with delayed response in growth and impulsive diffusion on nutrients:

$$
\begin{aligned}
& \frac{d s_{1}(t)}{d t}=D_{1}\left(s_{1}^{0}-s_{1}(t)\right), \\
& \frac{d s_{2}(t)}{d t}=D_{2}\left(s_{2}^{0}-s_{2}(t)\right)-P\left(s_{2}(t)\right) x(t), \quad t \neq n \tau, n=1,2 \ldots \\
& \frac{d x(t)}{d t}=-D_{2} x(t)+e^{-D_{2} \tau_{1}} P\left(s_{2}\left(t-\tau_{1}\right)\right) x\left(t-\tau_{1}\right),
\end{aligned}
$$




$$
\begin{gathered}
\Delta s_{1}(t)=d\left(s_{2}(t)-s_{1}(t)\right)+\mu_{1}, \\
\Delta s_{2}(t)=d\left(s_{1}(t)-s_{2}(t)\right), \quad t=n \tau, n=1,2 \ldots, \\
\Delta x(t)=0, \\
\left(\varphi_{1}(\zeta), \varphi_{2}(\zeta), \varphi_{3}(\zeta)\right) \in C_{+}=C\left(\left[-\tau_{1}, 0\right], R_{+}^{3}\right), \quad \varphi_{i}(0)>0, i=1,2,3,
\end{gathered}
$$

where we suppose that the system is composed of two lakes connected by impulsive diffusion. $s_{i}(t)$ denotes the concentration of the nutrient in $i$ th lake at time $t(i=1,2) \cdot s_{i}^{0}$ denotes the input nutrient concentration in $i$ th lake $(i=1,2) . D_{i}$ denotes the input rate from the lakes containing the substrate and the wash-out rate of substrate and microorganism by products from Lake $i(i=1,2) .0<d<1$ is diffusive rate between Lake 1 and Lake 2 . It is assumed here that the net exchange from patch $j$ to Lake $i$ is proportional to the difference $s_{j}-s_{i}$ of concentration of the nutrient. $x(t)$ denotes the concentration of the the microorganism in the second lake at time $t . \tau_{1}$ stands for the time delay in conversion of nutrient to biomass for the microorganism. As discussed in [20,32], $e^{-D_{2} \tau_{1}} x\left(t-\tau_{1}\right)$ represents the biomass of those microorganisms that consume nutrient $\tau_{1}$ units of time prior to time $t$ and that survive in the chemostat the $\tau_{1}$ units of time necessary to complete the nutrient conversion process. $P\left(s_{2}(t)\right)$ indicates the consumption rate of nutrient by the microorganism. It is assumed that $P(0)=$ $0, P^{\prime}(0)>0$ and $P^{\prime \prime}(0) \leq 0$. In particular, these hypotheses are satisfied by the MichaelisMenten function $P\left(s_{2}(t)\right)=s_{2}(t) /\left(k+s_{2}(t)\right)$, here $k>0$ is the half-saturation constant or Michaelis-Menten constant. The impulsive diffusion occurs every $\tau$ period $(\tau>0)$, the system evolves from its initial state without being further affected by diffusion until the next pulse appears. $\Delta x=x\left(n \tau^{+}\right)-x(n \tau)$, and $x\left(n \tau^{+}\right)$represents the concentration of the plankton in the $i$ th lake immediately after the $n$th diffusion pulse at time $t=n \tau$, while $x(n \tau)$ represents the concentration of the microorganism in the $i$ th lake before the $n$th diffusion pulse at time $t=n \tau, n \in Z_{+} . \mu_{1} \geq 0$ is the amount of the substrate concentration pulse at $t=n \tau, n \in Z_{+}$ in first lake. The purpose of this paper is to prove that the system (2.1) has a microorganismextinction periodic solution, further, it is globally attractive, and also prove system (2.1) is permanent.

\section{The Lemmas}

The solution of (2.1), denoted by $X(t)=\left(s_{1}(t), s_{2}(t), x(t)\right)^{T}$, is a piecewise continuous function $X: R_{+} \rightarrow R_{+}^{3} . X(t)$ is continuous on $(n \tau,(n+1) \tau], n \in Z_{+}$and $X\left(n \tau^{+}\right)=\lim _{t \rightarrow n \tau^{+}} X(t)$ exists. Obviously the global existence and uniqueness of solutions of (2.1) is guaranteed by the smoothness properties of $f$, which denotes the mapping defined by right-side of system (2.1) [1]. Before having the main results. we need some lemmas which will be used in the next.

According to the biological meanings, it is assumed that $s_{1}(t) \geq 0, s_{2}(t) \geq 0$ and $x(t) \geq$ 0 . Now, we will show that all solutions of (2.1) are uniformly ultimately bounded.

Lemma 3.1. There exists a constant $M>0$ such that $s_{1}(t) \leq M, s_{2}(t) \leq M, x(t) \leq e^{-D \tau} M$, for each solution $\left(s_{1}(t), s_{2}(t), x(t)\right)$ of (2.1) with all $t$ large enough. 
Proof. Define $V(t)=s_{1}(t)+s_{2}(t)+e^{D_{2} \tau} x\left(t+\tau_{1}\right)$, and $D=\min \left\{D_{1}, D_{2}\right\}$. When $t \neq n \tau$, we have

$$
\begin{aligned}
D^{+} V(t)+D V(t) & =D_{1} s_{1}^{0}+D_{2} s_{2}^{0}-\left(D_{1}-D\right) s_{1}(t)-\left(D_{2}-D\right) s_{2}(t)-\left(D_{2}-D\right) e^{-D_{2} \tau} x(t+\tau), \\
& <D_{1} s_{1}^{0}+D_{2} s_{2}^{0} .
\end{aligned}
$$

When $t=n \tau, V\left(n \tau^{+}\right)=\mu_{1}$. By [1, lemma 2.2], for $t \in(n \tau,(n+1) \tau]$, we have

$$
\begin{aligned}
V(t) \leq & V(0) \exp (-D t)+\int_{0}^{t}\left(D_{1} s_{1}^{0}+D_{2} s_{2}^{0}\right) \exp (-D(t-s)) d s+\sum_{0<n \tau<t} \mu_{1} \exp (-D(t-n \tau)) \\
= & V(0) \exp (-D t)+\frac{D_{1} s_{1}^{0}+D_{2} s_{2}^{0}}{D}(1-\exp (-D t)) \\
& +\mu_{1} \frac{\exp (-D(t-\tau))-\exp (-D(t-(n+1) \tau))}{1-\exp (D \tau)} \\
< & V(0) \exp (-D t)+\frac{D_{1} s_{1}^{0}+D_{2} s_{2}^{0}}{D}(1-\exp (-D t))+\frac{\mu_{1} \exp (-D(t-\tau))}{1-\exp (D \tau)}+\frac{\mu_{1} \exp (D \tau)}{\exp (D \tau)-1} \\
& \longrightarrow \frac{D_{1} s_{1}^{0}+D_{2} s_{2}^{0}}{D}+\frac{\mu_{1} \exp (D \tau)}{\exp (D \tau)-1}, \quad \text { as } t \longrightarrow \infty
\end{aligned}
$$

So $V(t)$ is uniformly ultimately bounded. Hence, by the definition of $V(t)$, there exists a constant $M>0$ such that $s_{1}(t) \leq M, s_{2}(t) \leq M, x(t) \leq e^{-D \tau} M$ for all $t$ large enough. The proof is complete.

If $x(t)=0$, the subsystem of $(2.1)$ is written as

$$
\begin{aligned}
& \frac{d s_{1}(t)}{d t}=D_{1}\left(s_{1}^{0}-s_{1}(t)\right), \\
& \frac{d s_{2}(t)}{d t}=D_{2}\left(s_{2}^{0}-s_{2}(t)\right), \\
& \Delta s_{1}(t)=d\left(s_{2}(t)-s_{1}(t)\right)+\mu_{1}, \quad t=n \tau, n=1,2 \ldots, \\
& \Delta s_{2}(t)=d\left(s_{1}(t)-s_{2}(t)\right),
\end{aligned}
$$
have

Integrating and solving the first two equations of system (3.3) between pulses, we

$$
s_{i}(t)=s_{i}^{0}-\left[s_{i}^{0}-s_{i}\left(n \tau^{+}\right)\right] e^{-D_{i}(t-n \tau)}, \quad n \tau<t \leq(n+1) \tau(i=1,2) .
$$


Considering the last two equations of system (3.3), we have the stroboscopic map of system (3.3) as follows:

$$
\begin{aligned}
s_{1}\left((n+1) \tau^{+}\right)= & (1-d) e^{-D_{1} \tau} s_{1}\left(n \tau^{+}\right)+d e^{-D_{2} \tau} s_{2}\left(n \tau^{+}\right) \\
& +(1-d)\left(1-e^{-D_{1} \tau}\right) s_{1}^{0}+d\left(1-e^{-D_{2} \tau}\right) s_{2}^{0}+\mu_{1}, \\
s_{2}\left((n+1) \tau^{+}\right)= & d e^{-D_{1} \tau} s_{1}\left(n \tau^{+}\right)+(1-d) e^{-D_{2} \tau} s_{2}\left(n \tau^{+}\right) \\
& +d\left(1-e^{-D_{1} \tau}\right) s_{1}^{0}+(1-d)\left(1-e^{-D_{2} \tau}\right) s_{2}^{0} .
\end{aligned}
$$

Equation (3.5) are difference equations. They describe the the concentration of the nutrients in the two lakes at a pulse in terms of values at the previous pulse. The dynamical behaviors of system (3.5) with equation (3.4) determine the dynamical behaviors of system (3.3). So we will devote to investigate system (3.5).

From system (3.5), we can easily have a unique fixed point of system (3.5) as follows

$$
\begin{aligned}
s_{1}^{*}= & \frac{\left[(1-d)\left(1-e^{-D_{1} \tau}\right)-(1-2 d)\left(1-e^{-D_{2} \tau}\right) e^{-D_{1} \tau}\right] s_{1}^{0}-d\left(1-e^{-D_{2} \tau}\right) s_{2}^{0}}{1-(1-d) e^{-D_{1} \tau}-(1-d) e^{-D_{2} \tau}-(1-2 d) e^{-\left(D_{1}+D_{2}\right) \tau}} \\
& +\frac{+\left(1-e^{-D_{2} \tau}+d e^{-D_{2} \tau}\right) \mu_{1}}{1-(1-d) e^{-D_{1} \tau}-(1-d) e^{-D_{2} \tau}-(1-2 d) e^{-\left(D_{1}+D_{2}\right) \tau}}, \\
s_{2}^{*}= & \frac{-d\left(1-e^{-D_{1} \tau}\right) s_{1}^{0}+\left[(1-d)\left(1-e^{-D_{2} \tau}\right)-(1-2 d)\left(1-e^{-D_{1} \tau}\right) e^{-D_{2} \tau}\right] s_{2}^{0}}{1-(1-d) e^{-D_{2} \tau}-(1-d) e^{-D_{1} \tau}-(1-2 d) e^{-\left(D_{2}+D_{1}\right) \tau}} \\
& +\frac{d e^{-D_{1} \tau} \mu_{1}}{1-(1-d) e^{-D_{2} \tau}-(1-d) e^{-D_{1} \tau}-(1-2 d) e^{-\left(D_{2}+D_{1}\right) \tau}},
\end{aligned}
$$

To write system (3.5) as a map, we define the map $F: R_{+}^{2} \rightarrow R_{+}^{2}$ :

$$
\begin{aligned}
F_{1}(x)= & (1-d) e^{-D_{1} \tau} x_{1}+d e^{-D_{2} \tau} x_{2} \\
& +(1-d)\left(1-e^{-D_{1} \tau}\right) s_{1}^{0}+d\left(1-e^{-D_{2} \tau}\right) s_{2}^{0}+\mu_{1} \\
F_{2}(x)= & d e^{-D_{1} \tau} x_{1}+(1-d) e^{-D_{2} \tau} x_{2} \\
& +d\left(1-e^{-D_{1} \tau}\right) s_{1}^{0}+(1-d)\left(1-e^{-D_{2} \tau}\right) s_{2}^{0} .
\end{aligned}
$$

$F(x)$ is the map evaluated at the point $x=\left(x_{1}, x_{2}\right) \in R_{+}^{2}$. Consequently, in system (3.4), $F^{n}$ describes the concentration of the nutrients in the time $n \tau$. 


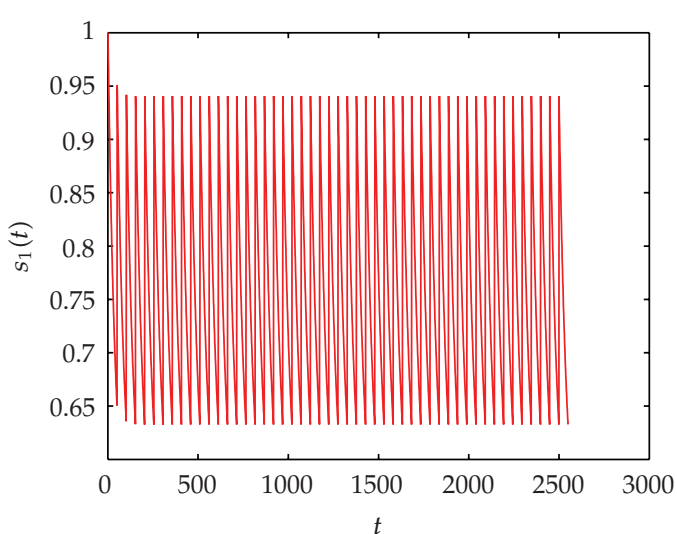

(a)

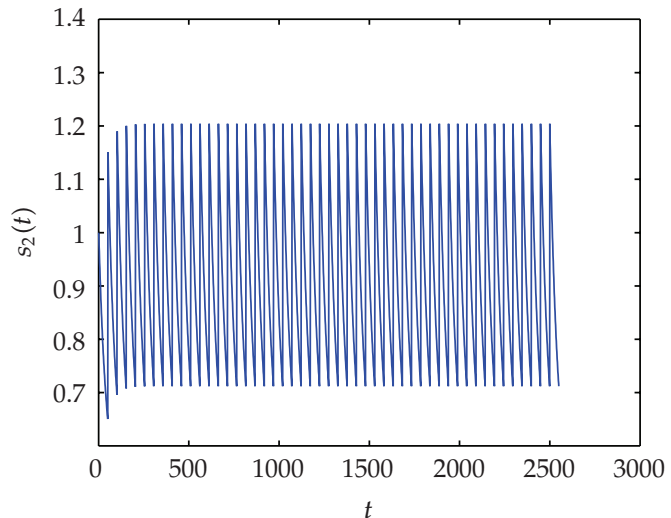

(b)

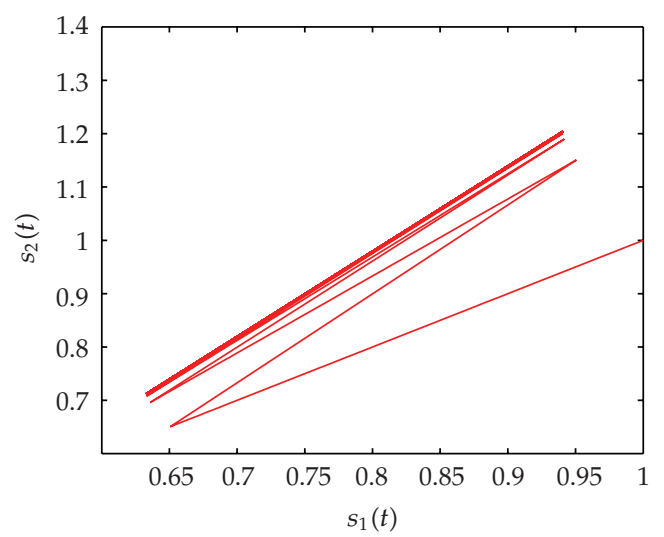

(c)

Figure 1: Dynamical behavior of system (3.3) with $s_{1}(0)=1, s_{2}(0)=1, s_{1}^{0}=0.5, s_{2}^{0}=0.5, D=0.6, d=0.1$, $\tau=2, \mu_{1}=0.3$. (a) time-series of $s_{1}(t)$, (b) time-series of $s_{2}(t)$, (c) The phase portrait of (3.3).

Doing similarly to [3, Lemma 4.1 and Theorem 4.1], and using iterative method, one can easily obtain Lemma 3.2 as follows:

Lemma 3.2. For every point $\left(x_{1}, x_{2}\right)>(0,0)$ of system (3.7), then one has

$$
F^{n}(x) \longrightarrow\left(s_{1}^{*}, s_{2}^{*}\right), \quad \text { as } n \longrightarrow \infty
$$

Remark 3.3. Lemma 3.2 implies that the fixed point $\left(s_{1}^{*}, s_{2}^{*}\right)$ of $F$ is globally stable. So all trajectories of (3.3) approach the positive periodic solution $\left.\widetilde{\left(s_{1}(t)\right.}, \widetilde{s_{2}(t)}\right)$ with period $\tau$, where $\widetilde{s_{i}(t)}=s_{i}^{0}-\left(s_{i}^{0}-s_{i}^{*}\right) e^{-D_{i}(t-n \tau)}, n \tau<t \leq(n+1) \tau,(i=1,2)$. The dynamical behaviors of system (3.3) also can be shown from Figure 1. 
Lemma 3.4 (see [18]). Consider the following delay equation:

$$
y^{\prime}(t)=a_{1} y(t-\tau)-a_{2} y(t)
$$

where $a_{1}, a_{2}, \tau>0 ; y(t)>0$ for $-\tau \leq t \leq 0$. If $a_{1}<a_{2}, \lim _{t \rightarrow \infty} y(t)=0$.

\section{Dynamical Behaviors of (2.1)}

From the above discussion, we know that there exists a microorganism-extinction boundary periodic solution $\left.\widetilde{\left(s_{1}(t)\right.}, \widetilde{s_{2}(t)}, 0\right)$ of system (2.1). In this section, we will prove that the microorganism-extinction boundary periodic solution $\left.\widetilde{\left(s_{1}(t)\right.}, \widetilde{s_{2}(t)}, 0\right)$ of system $(2.1)$ is globally attractive.

Theorem 4.1. If

$$
\begin{aligned}
& {\left[\frac{-d\left(1-e^{-D_{1} \tau}\right) s_{1}^{0}+\left[(1-d)\left(1-e^{-D_{2} \tau}\right)-(1-2 d)\left(1-e^{-D_{1} \tau}\right) e^{-D_{2} \tau}\right] s_{2}^{0}}{1-(1-d) e^{-D_{2} \tau}-(1-d) e^{-D_{1} \tau}-(1-2 d) e^{-\left(D_{2}+D_{1}\right) \tau}}\right.} \\
& \left.+\frac{d e^{-D_{1} \tau} \mu_{1}}{1-(1-d) e^{-D_{2} \tau}-(1-d) e^{-D_{1} \tau}-(1-2 d) e^{-\left(D_{2}+D_{1}\right) \tau}}\right] \\
& \quad<P^{-1}\left(D_{2} e^{D_{2} \tau_{1}}\right)
\end{aligned}
$$

holds, then the microorganism-extinction periodic solution $\left.\widetilde{s_{1}(t)}, \widetilde{s_{2}(t)}, 0\right)$ of system $(2.1)$ is globally attractive.

Proof. Since

$$
\begin{aligned}
& {\left[\frac{-d\left(1-e^{-D_{1} \tau}\right) s_{1}^{0}+\left[(1-d)\left(1-e^{-D_{2} \tau}\right)-(1-2 d)\left(1-e^{-D_{1} \tau}\right) e^{-D_{2} \tau}\right] s_{2}^{0}}{1-(1-d) e^{-D_{2} \tau}-(1-d) e^{-D_{1} \tau}-(1-2 d) e^{-\left(D_{2}+D_{1}\right) \tau}}\right.} \\
& \left.+\frac{d e^{-D_{1} \tau} \mu_{1}}{1-(1-d) e^{-D_{2} \tau}-(1-d) e^{-D_{1} \tau}-(1-2 d) e^{-\left(D_{2}+D_{1}\right) \tau}}\right] \\
& \quad<P^{-1}\left(D_{2} e^{D_{2} \tau_{1}}\right)
\end{aligned}
$$

then we can choose $\varepsilon_{0}$ sufficiently small such that

$$
\begin{aligned}
e^{-D_{2} \tau_{1}} P( & {\left[\frac{-d\left(1-e^{-D_{1} \tau}\right) s_{1}^{0}+\left[(1-d)\left(1-e^{-D_{2} \tau}\right)-(1-2 d)\left(1-e^{-D_{1} \tau}\right) e^{-D_{2} \tau}\right] s_{2}^{0}}{1-(1-d) e^{-D_{2} \tau}-(1-d) e^{-D_{1} \tau}-(1-2 d) e^{-\left(D_{2}+D_{1}\right) \tau}}\right.} \\
& \left.\left.+\frac{d e^{-D_{1} \tau} \mu_{1}}{1-(1-d) e^{-D_{2} \tau}-(1-d) e^{-D_{1} \tau}-(1-2 d) e^{-\left(D_{2}+D_{1}\right) \tau}}\right]+\varepsilon_{0}\right)<D_{2} .
\end{aligned}
$$


It follows from that the first equation of system (2.1) that $d s_{2}(t) / d t \leq D_{2}\left(s_{2}^{0}-s_{2}(t)\right)$. So we consider the following comparison impulsive differential system:

$$
\begin{gathered}
\frac{d x_{1}(t)}{d t}=D_{1}\left(s_{1}^{0}-x_{1}(t)\right), \\
\frac{d x_{2}(t)}{d t}=D_{2}\left(s_{2}^{0}-x_{2}(t)\right), \\
\Delta x_{1}(t)=d\left(x_{2}(t)-x_{1}(t)\right)+\mu_{1}, \\
\Delta x_{2}(t)=d\left(x_{1}(t)-x_{2}(t)\right), \\
x_{1}\left(0^{+}\right)=s_{1}\left(0^{+}\right), \\
x_{2}\left(0^{+}\right)=s_{2}\left(0^{+}\right) .
\end{gathered}
$$

In view of Remark 3.3, we obtain the periodic solution of system (4.4)

$$
\widetilde{x_{i}(t)}=s_{i}^{0}-\left(s_{i}^{0}-x_{i}^{*}\right) e^{-D_{i}(t-n \tau)}, \quad n \tau<t \leq(n+1) \tau \quad(i=1,2)
$$

which is globally asymptotically stable. Here

$$
\begin{aligned}
x_{1}^{*}= & \frac{\left[(1-d)\left(1-e^{-D_{1} \tau}\right)-(1-2 d)\left(1-e^{-D_{2} \tau}\right) e^{-D_{1} \tau}\right] s_{1}^{0}-d\left(1-e^{-D_{2} \tau}\right) s_{2}^{0}}{1-(1-d) e^{-D_{1} \tau}-(1-d) e^{-D_{2} \tau}-(1-2 d) e^{-\left(D_{1}+D_{2}\right) \tau}} \\
& +\frac{+\left(1-e^{-D_{2} \tau}+d e^{-D_{2} \tau}\right) \mu_{1}}{1-(1-d) e^{-D_{1} \tau}-(1-d) e^{-D_{2} \tau}-(1-2 d) e^{-\left(D_{1}+D_{2}\right) \tau}}, \\
x_{2}^{*}= & \frac{-d\left(1-e^{-D_{1} \tau}\right) s_{1}^{0}+\left[(1-d)\left(1-e^{-D_{2} \tau}\right)-(1-2 d)\left(1-e^{-D_{1} \tau}\right) e^{-D_{2} \tau}\right] s_{2}^{0}}{1-(1-d) e^{-D_{2} \tau}-(1-d) e^{-D_{1} \tau}-(1-2 d) e^{-\left(D_{2}+D_{1}\right) \tau}} \\
& +\frac{d e^{-D_{1} \tau} \mu_{1}}{1-(1-d) e^{-D_{2} \tau}-(1-d) e^{-D_{1} \tau}-(1-2 d) e^{-\left(D_{2}+D_{1}\right) \tau}} .
\end{aligned}
$$

From Remark 3.3 and comparison theorem of impulsive equation [1], we have $s_{i}(t) \leq$ $x_{i}(t)(i=1,2)$, and $x_{i}(t) \rightarrow \widetilde{s_{i}(t)}(i=1,2)$ as $t \rightarrow \infty$. Then there exists an integer $k_{2}^{\prime}>k_{1}$, $t>k_{2}^{\prime}$ such that

$$
s_{i}(t) \leq x_{i}(t) \leq \widetilde{s_{i}(t)}+\varepsilon_{0}, \quad n \tau<t \leq(n+1) \tau, n>k_{2}^{\prime},(i=1,2)
$$

that is,

$$
s_{2}(t)<\widetilde{s_{2}(t)}+\varepsilon_{0} \leq s_{2}^{*}+\varepsilon_{0} \stackrel{\Delta}{=} \rho, \quad n \tau<t \leq(n+1) \tau, n>k_{2}^{\prime}
$$

From the third equation of system (2.1), we get

$$
\frac{d x(t)}{d t} \leq e^{-D_{2} \tau_{1}} P(\rho) x\left(t-\tau_{1}\right)-D_{2} x(t), \quad t>n \tau+\tau_{1}, n>k_{2} .
$$


Considering the following comparison differential system:

$$
\frac{d y(t)}{d t}=e^{-D_{2} \tau_{1}} P(\rho) y\left(t-\tau_{1}\right)-D_{2} y(t), \quad t>n \tau+\tau_{1}, n>k_{2}
$$

we have $e^{-D_{2} \tau_{1}} P(\rho)<D_{2}$. According to Lemma 3.4, we have $\lim _{t \rightarrow \infty} y(t)=0$.

Let $\left(s_{1}(t), s_{2}(t), x(t)\right)$ be the solution of system (2.1) with initial conditions and $x(\zeta)=$ $\varphi_{3}(\zeta)\left(\zeta \in\left[-\tau_{1}, 0\right]\right), y(t)$ is the solution of system (4.10) with initial conditions $x(\zeta)=$ $\varphi_{3}(\zeta)\left(\zeta \in\left[-\tau_{1}, 0\right]\right)$. By the comparison theorem, we have

$$
\lim _{t \rightarrow \infty} x(t)<\lim _{t \rightarrow \infty} y(t)=0
$$

Incorporating into the positivity of $x(t)$, we know that $\lim _{t \rightarrow \infty} x(t)=0$, Therefore, for any $\varepsilon_{1}>0$ (sufficiently small), there exists an integer $k_{3}\left(k_{3} \tau>k_{2} \tau+\tau_{1}\right)$ such that $x(t)<\varepsilon_{1}$ for all $t>k_{3} \tau$.

For system (2.1), we have

$$
D_{2}\left(s_{2}^{0}-\left(1+\frac{\varepsilon_{1} P^{\prime}(0)}{D_{2}}\right) s_{2}(t)\right] \leq \frac{d s_{2}(t)}{d t} \leq D_{2}\left(s_{2}^{0}-s_{2}(t)\right)
$$

Then we have $z_{1}(t) \leq s_{1}(t) \leq z_{2}(t), z_{1}^{\prime}(t) \leq s_{2}(t) \leq z_{2}^{\prime}(t)$ and $z_{1}(t) \rightarrow \widetilde{s_{1}(t)}, z_{2}(t) \rightarrow \widetilde{s_{2}(t)}$, $z_{1}^{\prime}(t) \rightarrow \widetilde{s_{1}(t)}, z_{2}^{\prime}(t) \rightarrow \widetilde{s_{2}(t)}$ as $t \rightarrow \infty$. While $\left(z_{1}(t), z_{2}(t)\right)$ and $\left(z_{1}^{\prime}(t), z_{2}^{\prime}(t)\right)$ are the solutions of

$$
\begin{gathered}
\frac{d z_{1}(t)}{d t}=D_{1}\left(s_{1}^{0}-z_{1}(t)\right), \quad t \neq n \tau, \\
\frac{d z_{2}(t)}{d t}=D_{2}\left[s_{2}^{0}-\left(1+\frac{\varepsilon_{1} P^{\prime}(0)}{D_{2}}\right) z_{2}(t)\right], \quad t \neq n \tau, \\
z_{1}\left(t^{+}\right)=d\left(z_{2}(t)-z_{1}(t)\right)+\mu_{1}, \quad t=n \tau, \\
z_{2}\left(t^{+}\right)=d\left(z_{1}(t)-z_{2}(t)\right), \quad t=n \tau, \\
z_{1}\left(0^{+}\right)=s_{1}\left(0^{+}\right), \\
z_{2}\left(0^{+}\right)=s_{2}\left(0^{+}\right), \\
\frac{d z_{1}^{\prime}(t)}{d t}=D_{1}\left(s_{1}^{0}-z_{1}^{\prime}(t)\right), \quad t \neq n \tau, \\
\frac{d z_{2}^{\prime}(t)}{d t}=D_{2}\left(s_{2}^{0}-z_{2}^{\prime}(t)\right), \quad t \neq n \tau, \\
z_{1}\left(t^{+}\right)=d\left(z_{2}(t)-z_{1}(t)\right)+\mu_{1}, \quad t=n \tau, \\
z_{2}\left(t^{+}\right)=d\left(z_{1}(t)-z_{2}(t)\right), \quad t=n \tau, \\
z_{1}\left(0^{+}\right)=s_{1}\left(0^{+}\right), \\
z_{2}\left(0^{+}\right)=s_{2}\left(0^{+}\right),
\end{gathered}
$$


respectively. For $n \tau<t \leq(n+1) \tau, \widetilde{z_{1}(t)}=s_{1}^{0}-\left(s_{1}^{0}-z_{1}^{*}\right) e^{-D_{1}(t-n \tau)}$ and $\widetilde{z_{2}(t)}=D_{2} s_{2}^{0} /\left(D_{2}+\right.$ $\left.\varepsilon_{1} P^{\prime}(0)\right)-\left(D_{2} S_{2}^{0} /\left(D_{2}+\varepsilon_{1} P^{\prime}(0)\right)-z_{2}^{*}\right) e^{-\left(D_{2}+\varepsilon_{1} P^{\prime}(0)\right)(t-n \tau)}$, where

$$
\begin{aligned}
z_{1}^{*}= & \frac{\left[(1-d)\left(1-e^{-D_{1} \tau}\right)-(1-2 d)\left(1-e^{-\left(D_{2}+\varepsilon_{1} P^{\prime}(0)\right) \tau}\right) e^{-D_{1} \tau}\right] s_{1}^{0}-d\left(1-e^{-\left(D_{2}+\varepsilon_{1} P^{\prime}(0)\right) \tau}\right) p}{1-(1-d) e^{-D_{1} \tau}-(1-d) e^{-\left(D_{2}+\varepsilon_{1} P^{\prime}(0)\right) \tau}-(1-2 d) e^{-\left(D_{1}+D_{2}+\varepsilon_{1} P^{\prime}(0)\right) \tau}} \\
& +\frac{+\left(1-e^{-\left(D_{2}+\varepsilon_{1} P^{\prime}(0)\right) \tau}+d e^{-\left(D_{2}+\varepsilon_{1} P^{\prime}(0)\right) \tau}\right) \mu_{1}}{1-(1-d) e^{-D_{1} \tau}-(1-d) e^{-\left(D_{2}+\varepsilon_{1} P^{\prime}(0)\right) \tau}-(1-2 d) e^{-\left(D_{1}+D_{2}+\varepsilon_{1} P^{\prime}(0)\right) \tau}}, \\
z_{2}^{*}= & \frac{-d\left(1-e^{-D_{1} \tau}\right) s_{1}^{0}+\left[(1-d)\left(1-e^{-\left(D_{2}+\varepsilon_{1} P^{\prime}(0)\right) \tau}\right)-(1-2 d)\left(1-e^{-D_{1} \tau}\right) e^{-\left(D_{2}+\varepsilon_{1} P^{\prime}(0)\right) \tau}\right] p}{1-(1-d) e^{-\left(D_{2}+\varepsilon_{1} P^{\prime}(0)\right) \tau}-(1-d) e^{-D_{1} \tau}-(1-2 d) e^{-\left(D_{2}+\varepsilon_{1} P^{\prime}(0)+D_{1}\right) \tau}} \\
& +\frac{d e^{-D_{1} \tau} \mu_{1}}{1-(1-d) e^{-\left(D_{2}+\varepsilon_{1} P^{\prime}(0)\right) \tau}-(1-d) e^{-D_{1} \tau}-(1-2 d) e^{-\left(D_{2}+\varepsilon_{1} P^{\prime}(0)+D_{1}\right) \tau}},
\end{aligned}
$$

where $D$ denotes $D_{2} s_{2}^{0} /\left(D_{2}+\varepsilon_{1} P^{\prime}(0)\right)$. Therefore, for any $\varepsilon_{2}>0$. there exists a integer $k_{4}, n>$ $k_{4}$ such that $\widetilde{z_{1}(t)}-\varepsilon_{2}<s_{1}(t)<\widetilde{s_{1}(t)}+\varepsilon_{2}, \widetilde{z_{2}(t)}-\varepsilon_{2}<s_{2}(t)<\widetilde{s_{2}(t)}+\varepsilon_{2}$. Let $\varepsilon_{1} \rightarrow 0$, so we have $\widetilde{s_{1}(t)}-\varepsilon_{2}<s_{1}(t)<\widetilde{s_{1}(t)}+\varepsilon_{2}, \widetilde{s_{2}(t)}-\varepsilon_{2}<s_{2}(t)<\widetilde{s_{2}(t)}+\varepsilon_{2}$, for $t$ large enough, which implies $s_{1}(t) \rightarrow \widetilde{s_{1}(t)}, s_{2}(t) \rightarrow \widetilde{s_{2}(t)}$ as $t \rightarrow \infty$. This completes the proof.

Definition 4.2. System (2.1) is said to be permanent, if there are constants $m, M>0$ (independent of initial value) and a finite time $T_{0}$ such that for all solutions $\left(s_{1}(t), s_{2}(t), x(t)\right.$ ) with all initial values $s_{1}(t)>0, s_{2}(t)>0, x\left(0^{+}\right)>0, m \leq s_{1}(t)<M, m \leq s_{2}(t)<M$, $m \leq x(t) \leq e^{-D_{2} \tau} M$, holds for all $t \geq T_{0}$. Here $T_{0}$ may depend on the initial values $\left(s_{1}\left(0^{+}\right), s_{2}\left(0^{+}\right), x\left(0^{+}\right)\right)$.

Theorem 4.3. If

$$
\begin{aligned}
& {\left[\frac{-d\left(1-e^{-D_{1} \tau}\right) s_{1}^{0}+\left[(1-d)\left(1-e^{-\left(D_{2}+x^{*} P^{\prime}(0)\right) \tau}\right)-(1-2 d)\left(1-e^{-D_{1} \tau}\right) e^{-\left(D_{2}+x^{*} P^{\prime}(0)\right) \tau}\right] \mathcal{R}}{1-(1-d) e^{-\left(D_{2}+x^{*} P^{\prime}(0)\right) \tau}-(1-d) e^{-D_{1} \tau}-(1-2 d) e^{-\left(D_{2}+x^{*} P^{\prime}(0)+D_{1}\right) \tau}}\right.} \\
& \left.+\frac{d e^{-D_{1} \tau} \mu_{1}}{1-(1-d) e^{-\left(D_{2}+x^{*} P^{\prime}(0)\right) \tau}-(1-d) e^{-D_{1} \tau}-(1-2 d) e^{-\left(D_{2}+x^{*} P^{\prime}(0)+D_{1}\right) \tau}}\right] \\
& \quad>P^{-1}\left(D_{2} e^{D_{2} \tau_{1}}\right)
\end{aligned}
$$

where $R$ denotes $D_{2} s_{2}^{0} /\left(D_{2}+x^{*} P^{\prime}(0)\right)$, holds, then there is a positive constant $q$ such that each positive solution $\left(s_{1}(t), s_{2}(t), x(t)\right)$ of (2.1) satisfies $x(t) \geq q$, for t large enough. Where $x^{*}$ is determined by 
the following equation

$$
\begin{aligned}
& P\left[\frac{-d\left(1-e^{-D_{1} \tau}\right) s_{1}^{0}+\left[(1-d)\left(1-e^{-\left(D_{2}+x^{*} P^{\prime}(0)\right) \tau}\right)-(1-2 d)\left(1-e^{-D_{1} \tau}\right) e^{-\left(D_{2}+x^{*} P^{\prime}(0)\right) \tau}\right]}{1-(1-d) e^{-\left(D_{2}+x^{*} P^{\prime}(0)\right) \tau}-(1-d) e^{-D_{1} \tau}-(1-2 d) e^{-\left(D_{2}+x^{*} P^{\prime}(0)+D_{1}\right) \tau}}\right. \\
& \left.\quad+\frac{d e^{-D_{1} \tau} \mu_{1}}{1-(1-d) e^{-\left(D_{2}+x^{*} P^{\prime}(0)\right) \tau}-(1-d) e^{-D_{1} \tau}-(1-2 d) e^{-\left(D_{2}+x^{*} P^{\prime}(0)+D_{1}\right) \tau}}\right] \\
& \quad=D_{2} e^{D_{2} \tau_{1}}
\end{aligned}
$$

Proof. The third equation of system (2.1) can be rewritten as

$$
\frac{d x(t)}{d t}=\left[e^{-D_{2} \tau_{1}} P\left(s_{2}(t)\right)-D_{2}\right] x(t)-e^{-D_{2} \tau_{1}} \frac{d}{d t} \int_{t-\tau_{1}}^{t} P\left(s_{2}(u)\right) x(u) d u
$$

Let us consider any positive solution $\left(s_{1}(t), s_{2}(t), x(t)\right)$ of system (2.1). According to(4.14), $V(t)$ is defined as

$$
V(t)=x(t)+e^{-D_{2} \tau_{1}} \int_{t-\tau_{1}}^{t} P(s(u)) x(u) d u
$$

We calculate the derivative of $V(t)$ along the solution of (2.1):

$$
\frac{d V(t)}{d t}=\left[e^{-D_{2} \tau_{1}} P\left(s_{2}(t)\right)-D_{2}\right] x(t)
$$

Since

$$
\begin{aligned}
& {\left[\frac{-d\left(1-e^{-D_{1} \tau}\right) s_{1}^{0}+\left[(1-d)\left(1-e^{-\left(D_{2}+x^{*} P^{\prime}(0)\right) \tau}\right)-(1-2 d)\left(1-e^{-D_{1} \tau}\right) e^{-\left(D_{2}+x^{*} P^{\prime}(0)\right) \tau}\right] \mathcal{R}}{1-(1-d) e^{-\left(D_{2}+x^{*} P^{\prime}(0)\right) \tau}-(1-d) e^{-D_{1} \tau}-(1-2 d) e^{-\left(D_{2}+x^{*} P^{\prime}(0)+D_{1}\right) \tau}}\right.} \\
& \left.+\frac{d e^{-D_{1} \tau} \mu_{1}}{1-(1-d) e^{-\left(D_{2}+x^{*} P^{\prime}(0)\right) \tau}-(1-d) e^{-D_{1} \tau}-(1-2 d) e^{-\left(D_{2}+x^{*} P^{\prime}(0)+D_{1}\right) \tau}}\right] \\
& \quad>P^{-1}\left(D_{2} e^{D_{2} \tau_{1}}\right),
\end{aligned}
$$


then we can easily know that there exists sufficiently small $\varepsilon>0$ such that

$$
\begin{aligned}
& P\left[\frac{-d\left(1-e^{-D_{1} \tau}\right) s_{1}^{0}+\left[(1-d)\left(1-e^{-\left(D_{2}+x^{*} P^{\prime}(0)\right) \tau}\right)-(1-2 d)\left(1-e^{-D_{1} \tau}\right) e^{-\left(D_{2}+x^{*} P^{\prime}(0)\right) \tau}\right] \mathcal{R}}{1-(1-d) e^{-\left(D_{2}+x^{*} P^{\prime}(0)\right) \tau}-(1-d) e^{-D_{1} \tau}-(1-2 d) e^{-\left(D_{2}+x^{*} P^{\prime}(0)+D_{1}\right) \tau}}\right. \\
& \left.\quad+\frac{d e^{-D_{1} \tau} \mu_{1}}{1-(1-d) e^{-\left(D_{2}+x^{*} P^{\prime}(0)\right) \tau}-(1-d) e^{-D_{1} \tau}-(1-2 d) e^{-\left(D_{2}+x^{*} P^{\prime}(0)+D_{1}\right) \tau}}-\varepsilon\right] \\
& \quad>D_{2} e^{D_{2} \tau_{1}} .
\end{aligned}
$$

We claim that for any $t_{0}>0$, it is impossible that $x(t)<x^{*}$ for all $t>t_{0}$. Suppose that the claim is not valid. Then there is a $t_{0}>0$ such that $x(t)<x^{*}$ for all $t>t_{0}$. It follows from the second equation of (2.1) that for all $t>t_{0}$,

$$
\frac{d s_{2}(t)}{d t}>\left(D_{2}+x^{*} P^{\prime}(0)\right)\left[\frac{D_{2} s_{2}^{0}}{D_{2}+x^{*} P^{\prime}(0)}-s_{2}(t)\right] .
$$

Consider the following comparison impulsive system for all $t>t_{0}$ :

$$
\begin{gathered}
\frac{d v_{1}(t)}{d t}=D_{1}\left(s_{1}^{0}-v_{1}(t)\right), \quad t \neq n \tau \\
\frac{d v_{2}(t)}{d t}=\left(D_{2}+x^{*} P^{\prime}(0)\right)\left[\frac{D_{2} s_{2}^{0}}{D_{2}+x^{*} P^{\prime}(0)}-v_{2}(t)\right], \quad t \neq n \tau \\
\Delta v_{1}(t)=d\left(v_{2}(t)-v_{1}(t)\right)+\mu_{1}, \quad t=n \tau \\
\Delta v_{2}(t)=d\left(v_{1}(t)-v_{2}(t)\right), \quad t=n \tau \\
v_{1}\left(0^{+}\right)=s_{1}\left(0^{+}\right) \\
v_{2}\left(0^{+}\right)=s_{2}\left(0^{+}\right)
\end{gathered}
$$

By Lemma 3.5, we obtain $\widetilde{v_{1}(t)}=s_{1}^{0}-\left(s_{1}^{0}-v_{1}^{*}\right) e^{-D_{1}(t-n \tau)}, \widetilde{v_{2}(t)}=D_{2} s_{2}^{0} /\left(D_{2}+x^{*} P^{\prime}(0)\right)-$ $\left(D_{2} S_{2}^{0} /\left(D_{2}+x^{*} P^{\prime}(0)\right)-v_{2}^{*}\right) e^{-\left(D_{2}+x^{*} P^{\prime}(0)\right)(t-n \tau)}, n \tau<t \leq(n+1) \tau$, is the unique positive periodic solution of (4.22) which is globally asymptotically stable, where,

$$
\begin{aligned}
v_{1}^{*}= & \frac{\left[(1-d)\left(1-e^{-D_{1} \tau}\right)-(1-2 d)\left(1-e^{-\left(D_{2}+x^{*} P^{\prime}(0)\right) \tau}\right) e^{-D_{1} \tau}\right] s_{1}^{0}-d\left(1-e^{-\left(D_{2}+x^{*} P^{\prime}(0)\right) \tau}\right) \mathcal{R}}{1-(1-d) e^{-D_{1} \tau}-(1-d) e^{-\left(D_{2}+x^{*} P^{\prime}(0)\right) \tau}-(1-2 d) e^{-\left(D_{1}+D_{2}+x^{*} P^{\prime}(0)\right) \tau}} \\
& +\frac{+\left(1-e^{-\left(D_{2}+x^{*} P^{\prime}(0)\right) \tau}+d e^{-\left(D_{2}+x^{*} P^{\prime}(0)\right) \tau}\right) \mu_{1}}{1-(1-d) e^{-D_{1} \tau}-(1-d) e^{-\left(D_{2}+x^{*} P^{\prime}(0)\right) \tau}-(1-2 d) e^{-\left(D_{1}+D_{2}+x^{*} P^{\prime}(0)\right) \tau}}
\end{aligned}
$$




$$
\begin{aligned}
v_{2}^{*}= & \frac{-d\left(1-e^{-D_{1} \tau}\right) s_{1}^{0}+\left[(1-d)\left(1-e^{-\left(D_{2}+x^{*} P^{\prime}(0)\right) \tau}\right)-(1-2 d)\left(1-e^{-D_{1} \tau}\right) e^{-\left(D_{2}+x^{*} P^{\prime}(0)\right) \tau}\right] \mathcal{R}}{1-(1-d) e^{-\left(D_{2}+x^{*} P^{\prime}(0)\right) \tau}-(1-d) e^{-D_{1} \tau}-(1-2 d) e^{-\left(D_{2}+x^{*} P^{\prime}(0)+D_{1}\right) \tau}} \\
& +\frac{d e^{-D_{1} \tau} \mu_{1}}{1-(1-d) e^{-\left(D_{2}+x^{*} P^{\prime}(0)\right) \tau}-(1-d) e^{-D_{1} \tau}-(1-2 d) e^{-\left(D_{2}+x^{*} P^{\prime}(0)+D_{1}\right) \tau}} .
\end{aligned}
$$

By the comparison theorem for impulsive differential equation [1], we know that there exists $t_{1}\left(>t_{0}+\tau_{1}\right)$ such that the inequality $s_{2}(t) \geq v_{2}^{*}(t)-\varepsilon$ holds for $t \geq t_{1}$, thus $s_{2}(t) \geq v_{2}^{*}-\varepsilon$ for all $t \geq t_{1}$. For convenience, we make notation as $\sigma \triangleq v_{2}^{*}-\varepsilon$. So we have

$$
e^{-D_{2} \tau_{1}} P(\sigma)>D_{2}
$$

then we have

$$
V^{\prime}(t)>x(t)\left[e^{-D_{2} \tau_{1}} P(\sigma)-D_{2}\right]
$$

for all $t>t_{1}$. Set $x^{m}=\min _{t \in\left[t_{1}, t_{1}+\tau_{1}\right]} x(t)$, we will show that $x(t) \geq x^{m}$ for all $t \geq t_{1}$. Suppose the contrary, then there is a $T_{0}>0$ such that $x(t) \geq x^{m}$ for $t_{1} \leq t \leq t_{1}+\tau_{1}+T_{0}, x\left(t_{1}+\tau_{1}+T_{0}\right)=x^{m}$ and $x^{\prime}\left(t_{1}+\tau_{1}+T_{0}\right)<0$. Hence, the first equation of system (1.1) imply that

$$
\begin{aligned}
x^{\prime}\left(t_{1}+\tau_{1}+T_{0}\right) & =e^{-D_{2} \tau_{1}} P\left(s\left(t_{1}+\tau_{1}+T_{0}\right) x\left(t_{1}+\tau_{1}+T_{0}\right)-D_{2} x\left(t_{1}+\tau_{1}+T_{0}\right)\right), \\
& \geq\left[e^{-D_{2} \tau_{1}} P(\sigma)-D_{2}\right] x^{m}>0 .
\end{aligned}
$$

This is a contradiction. Thus, $x(t) \geq x^{m}$ for all $t>t_{1}$. As a consequence, Then $V^{\prime}(t)>$ $x^{m}\left[e^{-D_{2} \tau_{1}} P(\sigma)-D_{2}\right]>0$ for all $t>t_{1}$. This implies that as $t \rightarrow \infty, V(t) \rightarrow \infty$. It is a contradiction to $V(t) \leq M\left(1+\tau_{1} e^{-2 D_{2} \tau_{1}} P(M)\right)$. Hence, the claim is complete.

By the claim, we are left to consider two case. First, $x(t) \geq x^{*}$ for all $t$ large enough. Second, $x(t)$ oscillates about $x^{*}$ for $t$ large enough.

Define

$$
q=\min \left\{\frac{x^{*}}{2}, q_{1}\right\}
$$

where $q_{1}=x^{*} e^{-D_{2} \tau_{1}}$. We hope to show that $x(t) \geq q$ for all $t$ large enough. The conclusion is evident in first case. For the second case, let $t^{*}>0$ and $\xi>0$ satisfy $x\left(t^{*}\right)=x\left(t^{*}+\xi\right)=x^{*}$ and $x(t)<x^{*}$ for all $t^{*}<t<t^{*}+\xi$ where $t^{*}$ is sufficiently large such that $x(t)>\sigma$ for $t^{*}<t<t^{*}+\xi$, $x(t)$ is uniformly continuous. The positive solutions of (2.1) are ultimately bounded and $x(t)$ is not affected by impulses. Hence, there is a $T\left(0<t<\tau_{1}\right.$ and $T$ is dependent of the choice of $t^{*}$ ) such that $x\left(t^{*}\right)>x^{*} / 2$ for $t^{*}<t<t^{*}+T$. If $\xi<T$, there is nothing to prove. Let us consider the case $T<\xi<\tau_{1}$. Since $x^{\prime}(t)>-D_{2} x(t)$ and $x\left(t^{*}\right)=x^{*}$, it is clear that $x(t) \geq q_{1}$ for $t \in\left[t^{*}, t^{*}+\tau_{1}\right]$. Then, proceeding exactly as the proof for the above claim. We see that $x(t) \geq q_{1}$ for $t \in\left[t^{*}+\tau_{1}, t^{*}+\xi\right]$. Because the kind of interval $t \in\left[t^{*}, t^{*}+\xi\right]$ is chosen in an arbitrary way 

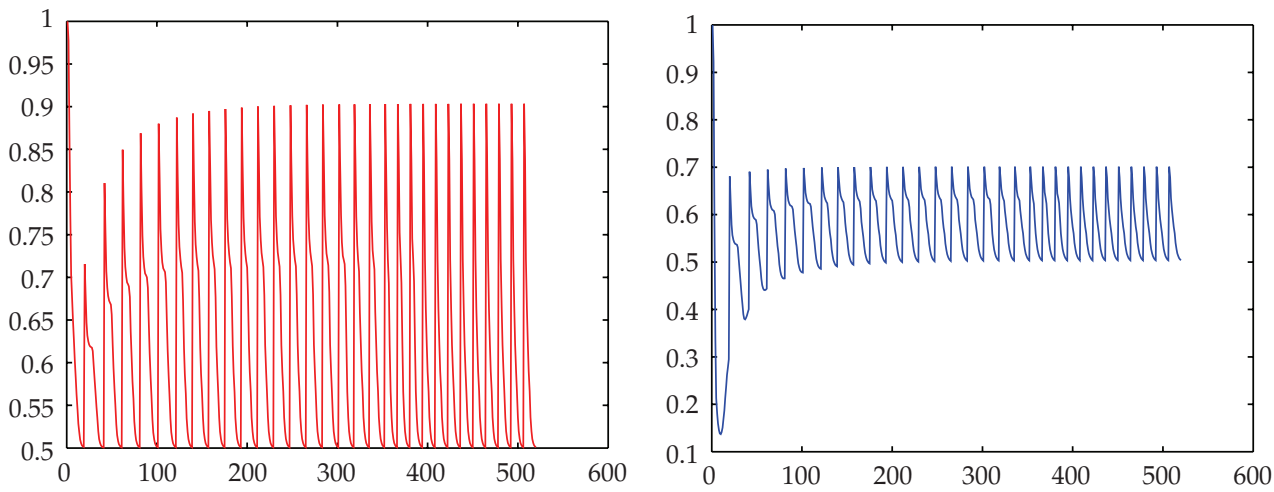

$-s_{1}(t)$

$-s_{2}(t)$

(a)

(b)

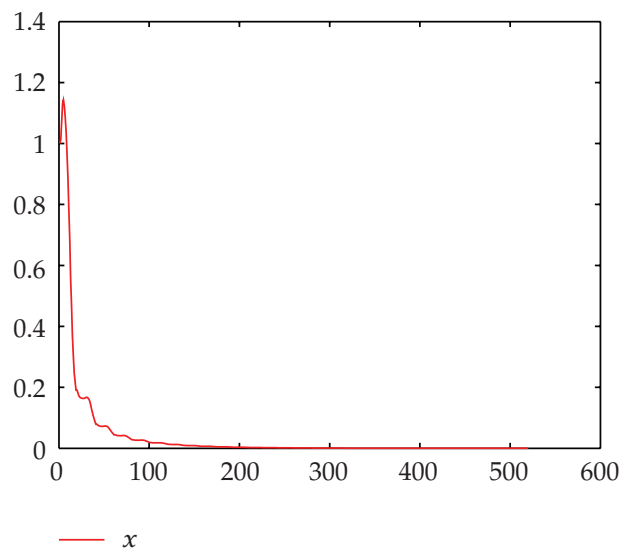

(c)

Figure 2: Dynamical behavior of system (2.1) on microorganism-extinction periodic solution with $s_{1}(0)=$ $1, s_{2}(0)=1, x(0)=1, s_{1}^{0}=0.5, s_{2}^{0}=0.5, D_{1}=0.6, D_{2}=0.4, k=2, \tau=1, \beta=2.5, \tau_{1}=1, d=0.9, \mu_{1}=0.4$. (a) time-series of $s_{1}(t),(\mathrm{b})$ time-series of $s_{2}(t),(\mathrm{c})$ time-series of $x(t)$.

(we only need $t^{*}$ to be large). We conclude $x(t) \geq q$ for all large $t$. In the second case. In view of above discussion, the choice of $q$ is independent of the positive solution, and we proved that any positive solution of (2.1) satisfies $x(t) \geq q$ for all sufficiently large $t$. This completes the proof of the theorem.

Theorem 4.4. If

$$
\begin{aligned}
& {\left[\frac{-d\left(1-e^{-D_{1} \tau}\right) s_{1}^{0}+\left[(1-d)\left(1-e^{-\left(D_{2}+x^{*} P^{\prime}(0)\right) \tau}\right)-(1-2 d)\left(1-e^{-D_{1} \tau}\right) e^{-\left(D_{2}+x^{*} P^{\prime}(0)\right) \tau}\right] \mathcal{R}}{1-(1-d) e^{-\left(D_{2}+x^{*} P^{\prime}(0)\right) \tau}-(1-d) e^{-D_{1} \tau}-(1-2 d) e^{-\left(D_{2}+x^{*} P^{\prime}(0)+D_{1}\right) \tau}}\right.} \\
& \left.+\frac{d e^{-D_{1} \tau} \mu_{1}}{1-(1-d) e^{-\left(D_{2}+x^{*} P^{\prime}(0)\right) \tau}-(1-d) e^{-D_{1} \tau}-(1-2 d) e^{-\left(D_{2}+x^{*} P^{\prime}(0)+D_{1}\right) \tau}}\right] \\
& \quad>P^{-1}\left(D_{2} e^{D_{2} \tau_{1}}\right),
\end{aligned}
$$

then system (2.1) is permanent. 


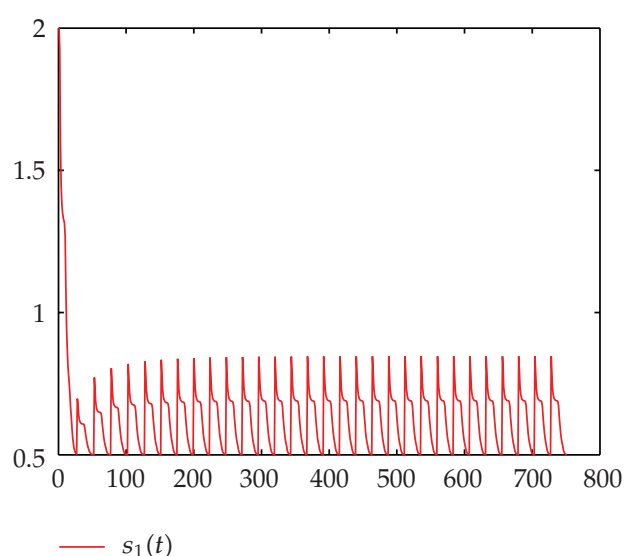

(a)

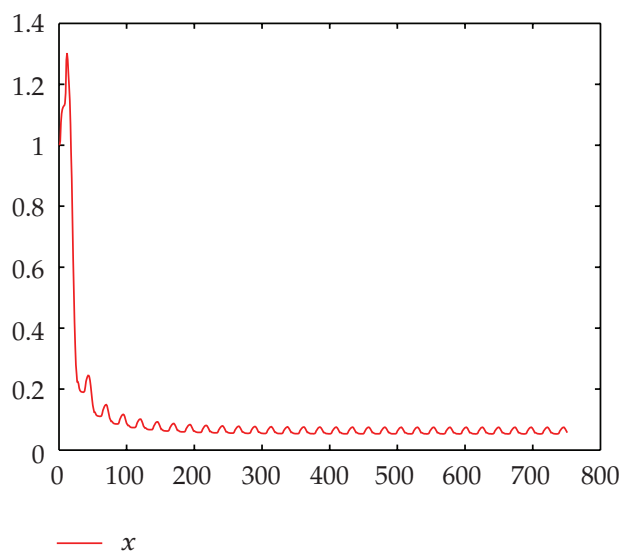

(c)

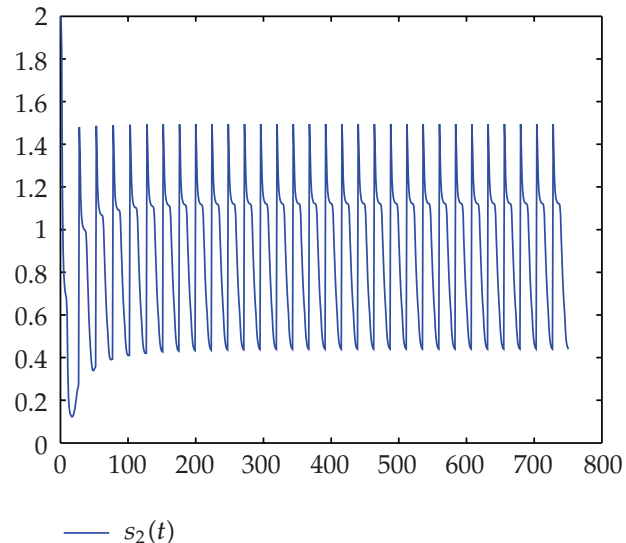

(b)

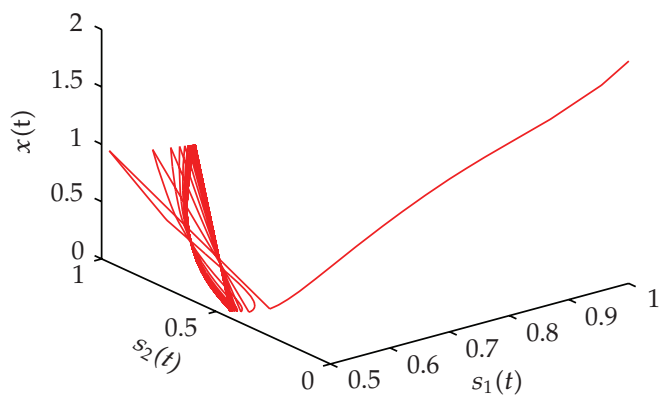

(d)

Figure 3: Dynamical behavior of the permanence of system $(2.1)$ with $s_{1}(0)=1, s_{2}(0)=1, x(0)=1$, $s_{1}^{0}=0.5, s_{2}^{0}=0.5, D_{1}=0.6, D_{2}=0.4, k=2, \tau=1, \beta=2.5, \tau_{1}=1, d=0.9, \mu_{1}=0.4$. (a) Time-series of $s_{1}(t)$. (b) Time-series of $s_{2}(t)$. (c) time-series of $x(t)$, (d) The phase portrait of the permanence of (2.1).

We can easily complete the proof of Theorem 4.4 by referring to Lemma 3.1, Theorems 4.1 and 4.3 .

\section{Discussion}

In this paper, we investigate a delayed chemostat model with impulsive diffusion and input on nutrients. From Theorems 4.1 and 4.4, we analyze that the microorganism-extinction periodic solution of system (2.1) is globally attractive, and System (2.1) is also proved to be permanent. If it is assumed that the consumption rate of nutrient by the plankton is $P\left(s_{2}(t)\right)=\beta s_{2}(t) /\left(k+s_{2}(t)\right)$, and $s_{1}(0)=1, s_{2}(0)=1, x(0)=1, s_{1}^{0}=0.5, s_{2}^{0}=0.5, D_{1}=0.6$, $D_{2}=0.4, k=2, \tau=1, \beta=2.5, \tau_{1}=1, d=0.9, \mu_{1}=0.4$. Then the microorganismextinction periodic solution is globally attractive (see Figure 2). If it is also assumed that the consumption rate of nutrient by the plankton is $P\left(s_{2}(t)\right)=\beta s_{2}(t) /\left(k+s_{2}(t)\right)$, and $s_{1}(0)=1$, $s_{2}(0)=1, x(0)=1, s_{1}^{0}=0.5, s_{2}^{0}=0.5, D_{1}=0.6, D_{2}=0.4, k=2, \tau=1, \beta=2.5, \tau_{1}=1$, 
$d=0.9, \mu_{1}=2$. then system (2.1) is permanence (see Figure 3). From Theorems 4.1 and 4.4, and the numerical analysis, we can easily guess that there must exist a threshold $\mu_{1}^{*}$. If $\mu_{1}<\mu_{1}^{*}$, the microorganism-extinction periodic solution $\left(s_{1}(t), s_{2}(t), 0\right)$ of $(2.1)$ is globally attractive. If $\mu_{1}>\mu_{1}^{*}$, system (2.1) is permanent. The sameness can be discussed on parameter $d$, then, the impulsive diffusion amount also plays an important role for the permanence of system (2.1). Our results provide strategy basis for biochemical reaction management.

\section{Acknowledgments}

This work was supported by National Natural Science Foundation of China (10961008), the Nomarch Foundation of Guizhou Province (2008035), the Science Technology Foundation of Guizhou (2008J2250), the Science Technology Foundation of Guizhou Education Department (2008038), and the National Key Technology R\&D Program of China (2006BAC01A09).

\section{References}

[1] D. BaĬnov and P. Simeonov, Impulsive Differential Equations: Periodic Solutions and Applications, vol. 66 of Pitman Monographs and Surveys in Pure and Applied Mathematics, Longman Scientific \& Technical, Harlow, UK, 1993.

[2] J. Jiao and L. Chen, "Global attractivity of a stage-structure variable coefficients predator-prey system with time delay and impulsive perturbations on predators," International Journal of Biomathematics, vol. 1, no. 2, pp. 197-208, 2008.

[3] L. Wang, Z. Liu, J. Hui, and L. Chen, "Impulsive diffusion in single species model," Chaos, Solitons and Fractals, vol. 33, no. 4, pp. 1213-1219, 2007.

[4] V. Lakshmikantham, D. D. BaĬnov, and P. S. Simeonov, Theory of Impulsive Differential Equations, vol. 6 of Series in Modern Applied Mathematics, World Scientific, Teaneck, NJ, USA, 1989.

[5] M. Benchohra, J. Henderson, and S. Ntouyas, Impulsive Differential Equations and Inclusions, vol. 2 of Contemporary Mathematics and Its Applications, Hindawi Publishing Corporation, New York, NY, USA, 2006.

[6] A. M. Samoǐlenko and N. A. Perestyuk, Impulsive Differential Equations, vol. 14 of World Scientific Series on Nonlinear Science. Series A: Monographs and Treatises, World Scientific, River Edge, NJ, USA, 1995.

[7] S. T. Zavalishchin and A. N. Sesekin, Dynamic Impulse Systems. Theory and Application, vol. 394 of Mathematics and Its Applications, Kluwer Academic Publishers, Dordrecht, The Netherlands, 1997.

[8] J. Jiao, L. Chen, and S. Cai, "An SEIRS epidemic model with two delays and pulse vaccination," Journal of Systems Science E Complexity, vol. 21, no. 2, pp. 217-225, 2008.

[9] G. Zeng, F. Wang, and J. J. Nieto, "Complexity of a delayed predator-prey model with impulsive harvest and Holling type II functional response," Advances in Complex Systems, vol. 11, no. 1, pp. 7797, 2008.

[10] H. Zhang, L. Chen, and J. J. Nieto, "A delayed epidemic model with stage-structure and pulses for pest management strategy," Nonlinear Analysis: Real World Applications, vol. 9, no. 4, pp. 1714-1726, 2008.

[11] W. Wang, J. Shen, and J. J. Nieto, "Permanence and periodic solution of predator-prey system with Holling type functional response and impulses," Discrete Dynamics in Nature and Society, vol. 2007, Article ID 81756, 15 pages, 2007.

[12] J. Jiao and L. Chen, "Dynamical analysis of a chemostat model with delayed response in growth and pulse input in polluted environment," Journal of Mathematical Chemistry, vol. 46, no. 2, pp. 502-513, 2009.

[13] X. Meng, Z. Li, and J. J. Nieto, "Dynamic analysis of Michaelis-Menten chemostat-type competition models with time delay and pulse in a polluted environment," Journal of Mathematical Chemistry, vol. 47, no. 1, pp. 123-144, 2009.

[14] J. Jiao, X. Yang, L. Chen, and S. Cai, "Effect of delayed response in growth on the dynamics of a chemostat model with impulsive input," Chaos, Solitons and Fractals, vol. 42, no. 4, pp. 2280-2287, 2009. 
[15] O. Tagashira, "Permanent coexistence in chemostat models with delayed feedback control," Nonlinear Analysis: Real World Applications, vol. 10, no. 3, pp. 1443-1452, 2009.

[16] H. L. Smith and P. Waltman, The Theory of the Chemostat. Dynamics of Microbial Competition, vol. 13 of Cambridge Studies in Mathematical Biology, Cambridge University Press, Cambridge, UK, 1995, .

[17] H. L. Smith and P. Waltman, "Perturbation of a globally stable steady state," Proceedings of the American Mathematical Society, vol. 127, no. 2, pp. 447-453, 1999.

[18] Y. Kuang, Delay Differential Equations with Applications in Population Dynamics, vol. 191 of Mathematics in Science and Engineering, Academic Press, Boston, Mass, USA, 1993.

[19] H. I. Freedman, J. W.-H. So, and P. Waltman, "Chemostat competition with time delays," in Biomedical Systems Modelling and Simulation (Paris, 1988), vol. 5 of IMACS Annals of Computing and Applied Mathematics, pp. 171-173, Baltzer, Basel, Switzerland, 1989.

[20] S. F. Ellermeyer, "Competition in the chemostat: global asymptotic behavior of a model with delayed response in growth," SIAM Journal on Applied Mathematics, vol. 54, no. 2, pp. 456-465, 1994.

[21] L. E. Caltagirone and R. L. Doutt, "The history of the vedalia beetle importation to California and its impact on the development of biological control," Annual Review of Entomology, vol. 34, pp. 1-16, 1989.

[22] S.-B. Hsu, P. Waltman, and S. F. Ellermeyer, "A remark on the global asymptotic stability of a dynamical system modeling two species competition," Hiroshima Mathematical Journal, vol. 24, no. 2, pp. 435-445, 1994.

[23] J. K. Hale and A. S. Somolinos, "Competition for fluctuating nutrient," Journal of Mathematical Biology, vol. 18, no. 3, pp. 255-280, 1983.

[24] S. B. Hsu, S. Hubbell, and P. Waltman, "A mathematical theory for single-nutrient competition in continuous cultures of micro-organisms," SIAM Journal on Applied Mathematics, vol. 32, no. 2, pp. 366 383, 1977.

[25] G. S. K. Wolkowicz and X.-Q. Zhao, "N-species competition in a periodic chemostat," Differential and Integral Equations, vol. 11, no. 3, pp. 465-491, 1998.

[26] S. Ellermeyer, J. Hendrix, and N. Ghoochan, "A theoretical and empirical investigation of delayed growth response in the continuous culture of bacteria," Journal of Theoretical Biology, vol. 222, no. 4, pp. 485-494, 2003.

[27] A. W. Bush and A. E. Cook, "The effect of time delay and growth rate inhibition in the bacterial treatment of wastewater," Journal of Theoretical Biology, vol. 63, no. 2, pp. 385-395, 1975.

[28] S. A. Levin and L. A. Segel, "Hypothesis for origin of planktonic patchiness," Nature, vol. 259, no. 5545, p. 659, 1976.

[29] M. Mimura, "Asymptotic behaviors of a parabolic system related to a planktonic prey and predator model," SIAM Journal on Applied Mathematics, vol. 37, no. 3, pp. 499-512, 1979.

[30] A. Okubo, Diffusion and Ecological Problems: Mathematical Models, vol. 10 of Biomathematics, Springer, Berlin, Germany, 1980.

[31] H. I. Freedman and S. Ruan, “On reaction-diffusion systems of zooplankton-phytoplankton-nutrient models," Differential Equations and Dynamical Systems, vol. 2, no. 1, pp. 49-64, 1994.

[32] S. Ruan, "Turing instability and travelling waves in diffusive plankton models with delayed nutrient recycling," IMA Journal of Applied Mathematics, vol. 61, no. 1, pp. 15-32, 1998.

[33] E. Funasaki and M. Kot, "Invasion and chaos in a periodically pulsed mass-action chemostat," Theoretical Population Biology, vol. 44, no. 2, pp. 203-224, 1993.

[34] R. J. Smith and G. S. K. Wolkowicz, "Analysis of a model of the nutrient driven self-cycling fermentation process," Dynamics of Continuous, Discrete $\mathcal{E}$ Impulsive Systems. Series B, vol. 11, no. 3, pp. 239-265, 2004. 